\title{
Variations in microbial composition and function in metabolic diseases
}

\section{obesity was \\ associated \\ with specific \\ gut microbial \\ taxa and \\ functions, \\ whereas \\ T2DM had \\ only a weak \\ association}

The gut microbiome is known to be dysregulated in metabolic disease. However, as obesity and type 2 diabetes mellitus (T2DM) often occur together, teasing out the changes that are specific to each condition has been challenging. A new study published in Cell Host \& Microbe aims to address this issue, and has identified a range of changes in the gut microbiota that are associated with either obesity or T2DM.

The researchers analysed the gut microbiomes of 633 lean individuals $\left(\mathrm{BMI} \leq 25 \mathrm{~kg} / \mathrm{m}^{2}\right)$ who did not have T2DM, 494 individuals with obesity $\left(\mathrm{BMI}>30 \mathrm{~kg} / \mathrm{m}^{2}\right)$ but not T2DM and 153 individuals with obesity and diagnosed T2DM or fasting plasma levels of glucose $>125 \mathrm{mg} / \mathrm{dl}$. Individuals with irritable bowel syndrome or inflammatory bowel disease were excluded from the study. The study participants were from population and metabolic disease cohorts in Germany (PopGen and FoCus). As well as assessing the composition of the gut

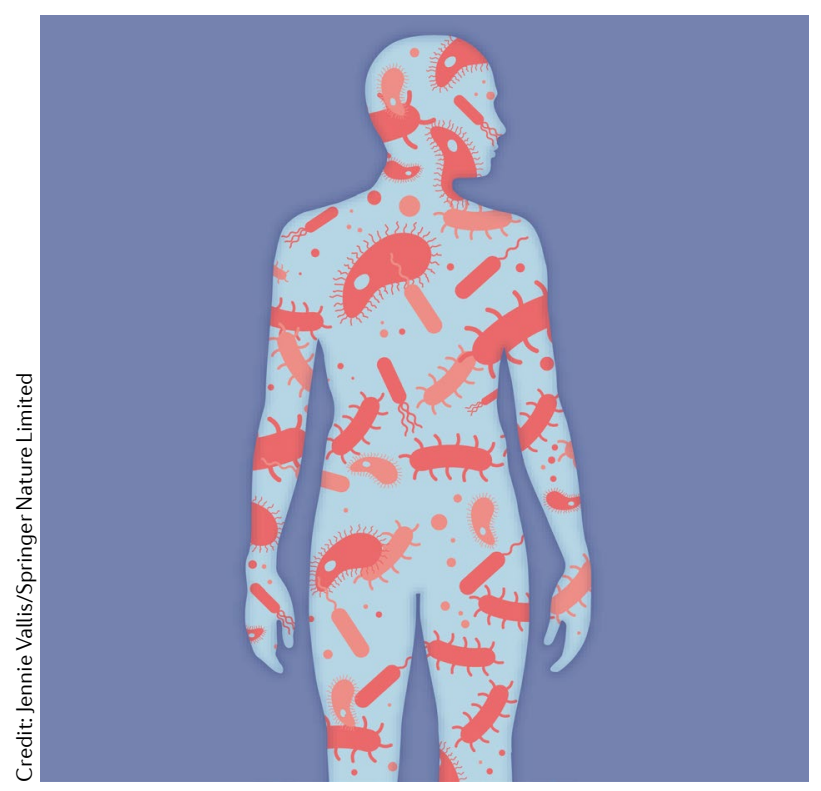

microbiomes of the participants, the researchers analysed their medical histories, serum metabolomics, biometrics and dietary data. Key findings were also replicated in 880 individuals from the SHIP cohort.

The analyses revealed that obesity was associated with specific gut microbial taxa and functions, whereas T2DM had only a weak association with specific gut microbial taxa. The researchers found that changes in 17 genera were specific to obesity, including decreased abundance of Akkermansia, Faecalibacterium, Oscillibacter and Alistipes. Faecalibacterium prausnitzii, which is usually abundant and has anti-inflammatory effects, was reduced in participants with only obesity, but was not associated with T2DM. They also noted small increases in Escherichia and Shigella that were associated with T2DM but not obesity.

Further analyses of pathways and gene families revealed that the gut microbiome of the participants with obesity but not T2DM had a reduced capacity for unidirectional conjugation (transfer of genetic material between bacteria). This finding indicates that the levels of reactive oxygen species are probably increased in the intestine of people with obesity. Interestingly, there was little difference between the pathways and gene families in the participants with obesity with or without T2DM.

The researchers also tested the effects of five medication classes (analgesics, antidiabetics, antidepressants, antiphlogistics and antihypertensives), use of dietary supplements and diet on gut microbial composition and function. The analysis showed that while medications and dietary supplements influenced the composition of the gut microbiota, an evaluation of specific taxa and microbial processes was unable to identify specific variation associated with medications and supplements. However, the researchers note that their study might not have been adequately powered to detect these differences.

Evaluation of dietary patterns (using food frequency questionnaires) showed that diet influenced the gut microbial composition and function in the participants without T2DM; however, long-term dietary factors could only account for a small proportion of the variance. The authors note that although the effects of individual medications, supplements and dietary factors on the gut microbiota are weak, in aggregate, these factors account for a considerable amount of the gut microbial variation in the study.

Finally, the investigators assessed the effects of obesity and T2DM on serum metabolite profiles. The overall analysis showed that both obesity and T2DM were associated with the overall serum metabolite composition. For instance, glutamate was increased in participants with obesity. In addition, levels of 3-hydroxyoctanoate were higher in people with obesity and T2DM than those with just obesity and lower in people with only obesity than in those who were lean.

The authors conclude that their paper adds to the growing body of literature detailing the association between the gut microbiome and obesity. They indicate that more work is needed to better define the effects of host-microbiome interactions in metabolic diseases.

Claire Greenhill

ORIGINAL ARTICLE Thingholm, L. B. et al. Obese individuals with and without type 2 diabetes show different gut microbial functional capacity and composition. Cell Host Microbe https://doi.org/ 10.1016/j.chom.2019.07.004 (2019) 